\title{
List of participants
}

\begin{tabular}{|l|l|l|l|l|}
\hline $\begin{array}{l}\text { Participant's } \\
\text { pseudonym }\end{array}$ & Archive ID number & $\begin{array}{l}\text { Year of } \\
\text { birth }\end{array}$ & Gender & Collection \\
\hline Seamus & LHArchiveA02 & 1916 & Male & LHSC Cohort 1 \\
\hline Joan & LHArchiveA03 & 1916 & Female & LHSC Cohort 1 \\
\hline Evelyn & LHArchiveA06 & 1923 & Female & LHSC Cohort 1 \\
\hline Rebecca & LHArchiveA07 & 1923 & Female & LHSC Cohort 1 \\
\hline James & LHArchiveA09 & 1924 & Male & LHSC Cohort 1 \\
\hline Kathleen & LHArchiveA11 & 1924 & Female & LHSC Cohort 1 \\
\hline Clifford & LHArchiveA13 & 1926 & Male & LHSC Cohort 1 \\
\hline Ben & LHArchiveA14 & 1926 & Male & LHSC Cohort 1 \\
\hline Rosemary & LHArchiveA16 & 1927 & Female & LHSC Cohort 1 \\
\hline Irene & LHArchiveA19 & 1928 & Female & LHSC Cohort 1 \\
\hline Luke & LHArchiveA20 & 1928 & Male & LHSC Cohort 1 \\
\hline Peter & LHArchiveA21 & 1928 & Male & LHSC Cohort 1 \\
\hline Eileen & LHArchiveA22 & 1928 & Female & LHSC Cohort 1 \\
\hline Simon & LHArchiveA23 & 1928 & Male & LHSC Cohort 1 \\
\hline Laura & LHArchiveA24 & 1928 & Female & LHSC Cohort 1 \\
\hline Mary & LHArchiveA25 & 1929 & Female & LHSC Cohort 1 \\
\hline William & LHArchiveA26 & 1930 & Male & LHSC Cohort 1 \\
\hline Lucy & LHArchiveA27 & 1930 & Female & LHSC Cohort 1 \\
\hline Joseph & LHArchiveA28 & 1930 & Male & LHSC Cohort 1 \\
\hline Deirdre & LHArchiveA29 & 1930 & Female & LHSC Cohort 1 \\
\hline Nancy & LHArchiveA30 & 1930 & Female & LHSC Cohort 1 \\
\hline Claire & LHArchiveA31 & 1931 & Female & LHSC Cohort 1 \\
\hline & & & & \\
\hline
\end{tabular}




\begin{tabular}{|c|c|c|c|c|}
\hline $\begin{array}{l}\text { Participant's } \\
\text { pseudonym }\end{array}$ & Archive ID number & $\begin{array}{l}\text { Year of } \\
\text { birth }\end{array}$ & Gender & Collection \\
\hline Graham & LHArchiveA32 & 1931 & Male & LHSC Cohort 1 \\
\hline Nuala & LHArchiveA33 & 1931 & Female & LHSC Cohort 1 \\
\hline Lillian & LHArchiveA34 & 1932 & Female & LHSC Cohort 1 \\
\hline Rita & LHArchiveA35 & 1932 & Female & LHSC Cohort 1 \\
\hline Monica & LHArchiveA36 & 1932 & Female & LHSC Cohort 1 \\
\hline Mark & LHArchiveA37 & 1933 & Male & LHSC Cohort 1 \\
\hline Clara & LHArchiveA38 & 1933 & Female & LHSC Cohort 1 \\
\hline Patricia & LHArchiveA39 & 1933 & Female & LHSC Cohort 1 \\
\hline Andrew & LHArchiveA40 & 1934 & Male & LHSC Cohort 1 \\
\hline Delores & LHArchiveA41 & 1934 & Female & LHSC Cohort 1 \\
\hline Patrick & LHArchiveA42 & 1934 & Male & LHSC Cohort 1 \\
\hline Tony & LHArchiveA43 & 1934 & Male & LHSC Cohort 1 \\
\hline Declan & LHArchiveA44 & 1934 & Male & LHSC Cohort 1 \\
\hline Liam & LHArchiveA45 & 1934 & Male & LHSC Cohort 1 \\
\hline Brenda & LHArchiveA46 & 1934 & Male & LHSC Cohort 1 \\
\hline Doreen & LHArchiveB01 & 1945 & Female & LHSC Cohort 2 \\
\hline David & LHArchiveB02 & 1945 & Male & LHSC Cohort 2 \\
\hline Audrey & LHArchiveB03 & 1945 & Female & LHSC Cohort 2 \\
\hline Denise & LHArchiveB04 & 1945 & Female & LHSC Cohort 2 \\
\hline Desmond & LHArchiveB05 & 1945 & Male & LHSC Cohort 2 \\
\hline Jillian & LHArchiveB06 & 1945 & Female & LHSC Cohort 2 \\
\hline Linda & LHArchiveB07 & $1950 \mathrm{~s}$ & Female & LHSC Cohort 2 \\
\hline Anthony & LHArchiveB08 & 1946 & Male & LHSC Cohort 2 \\
\hline Geoff & LHArchiveB09 & 1946 & Male & LHSC Cohort 2 \\
\hline Brigit & LHArchiveB10 & 1946 & Female & LHSC Cohort 2 \\
\hline Daniel & LHArchiveB11 & 1947 & Male & LHSC Cohort 2 \\
\hline Sarah & LHArchiveB12 & 1946 & Female & LHSC Cohort 2 \\
\hline Phyllis & LHArchiveB13 & 1946 & Female & LHSC Cohort 2 \\
\hline $\mathrm{Liz}$ & LHArchiveB15 & 1946 & Female & LHSC Cohort 2 \\
\hline Owen & LHArchiveB16 & 1946 & Male & LHSC Cohort 2 \\
\hline John & LHArchiveB17 & 1946 & Male & LHSC Cohort 2 \\
\hline Jack & LHArchiveB18 & 1947 & Male & LHSC Cohort 2 \\
\hline Judith & LHArchiveB20 & 1946 & Female & LHSC Cohort 2 \\
\hline Betty & LHArchiveB21 & 1947 & Female & LHSC Cohort 2 \\
\hline
\end{tabular}




\begin{tabular}{|c|c|c|c|c|}
\hline $\begin{array}{l}\text { Participant's } \\
\text { pseudonym }\end{array}$ & Archive ID number & $\begin{array}{l}\text { Year of } \\
\text { birth }\end{array}$ & Gender & Collection \\
\hline Martin & LHArchiveB22 & 1947 & Male & LHSC Cohort 2 \\
\hline Maire & LHArchiveB23 & 1949 & Female & LHSC Cohort 2 \\
\hline Vincent & LHArchiveB24 & 1949 & Male & LHSC Cohort 2 \\
\hline Sally & LHArchiveB25 & 1949 & Female & LHSC Cohort 2 \\
\hline Siobhan & LHArchiveB26 & 1950 & Female & LHSC Cohort 2 \\
\hline Edward & LHArchiveB27 & 1950 & Male & LHSC Cohort 2 \\
\hline Nora & LHArchiveB28 & 1950 & Female & LHSC Cohort 2 \\
\hline Enda & LHArchiveB29 & 1950 & Male & LHSC Cohort 2 \\
\hline Bernard & LHArchiveB14 & 1951 & Male & LHSC Cohort 2 \\
\hline Darren & LHArchiveB30 & 1951 & Male & LHSC Cohort 2 \\
\hline Sharon & LHArchiveB31 & 1951 & Female & LHSC Cohort 2 \\
\hline Rose & LHArchiveB32 & $1950 \mathrm{~s}$ & Female & LHSC Cohort 2 \\
\hline Pauleen & LHArchiveB33 & 1952 & Female & LHSC Cohort 2 \\
\hline Sean & LHArchiveB34 & 1952 & Male & LHSC Cohort 2 \\
\hline Francis & LHArchiveB35 & 1952 & Male & LHSC Cohort 2 \\
\hline Susan & LHArchiveB36 & 1953 & Female & LHSC Cohort 2 \\
\hline Anne & LHArchiveB37 & 1953 & Female & LHSC Cohort 2 \\
\hline Rob & LHArchiveB38 & 1953 & Male & LHSC Cohort 2 \\
\hline Cormac & LHArchiveB39 & 1954 & Male & LHSC Cohort 2 \\
\hline Barney & LHArchiveB41 & 1954 & Male & LHSC Cohort 2 \\
\hline Marguerite & LHArchiveB42 & 1952 & Female & LHSC Cohort 2 \\
\hline Marion & LHArchiveC02 & 1965 & Female & LHSC Cohort 3 \\
\hline Mandy & LHArchiveC03 & 1965 & Female & LHSC Cohort 3 \\
\hline Chris & LHArchiveC04 & 1965 & Male & LHSC Cohort 3 \\
\hline Anne-Marie & LHArchiveC05 & 1966 & Female & LHSC Cohort 3 \\
\hline Brian & LHArchiveC06 & 1966 & Male & LHSC Cohort 3 \\
\hline Orla & LHArchiveC07 & 1966 & Female & LHSC Cohort 3 \\
\hline Kevin & LHArchiveC08 & 1967 & Male & LHSC Cohort 3 \\
\hline Niamh & LHArchiveC09 & $1960 \mathrm{~s}$ & Female & LHSC Cohort 3 \\
\hline Aine & LHArchiveC11 & 1969 & Female & LHSC Cohort 3 \\
\hline Lorraine & LHArchiveC12 & 1969 & Female & LHSC Cohort 3 \\
\hline Elizabeth & LHArchiveC13 & 1970 & Female & LHSC Cohort 3 \\
\hline Ruth & LHArchiveC14 & $1970 \mathrm{~s}$ & Female & LHSC Cohort 3 \\
\hline Donal & LHArchiveC15 & 1970 & Male & LHSC Cohort 3 \\
\hline
\end{tabular}




\begin{tabular}{|c|c|c|c|c|}
\hline $\begin{array}{l}\text { Participant's } \\
\text { pseudonym }\end{array}$ & Archive ID number & $\begin{array}{l}\text { Year of } \\
\text { birth }\end{array}$ & Gender & Collection \\
\hline Grace & withheld & $1970 \mathrm{~s}$ & Female & LHSC Cohort 3 \\
\hline Louise & LHArchiveC17 & 1970 & Female & LHSC Cohort 3 \\
\hline Emer & LHArchiveC19 & $1970 \mathrm{~s}$ & Female & LHSC Cohort 3 \\
\hline Thomas & LHArchiveC20 & 1972 & Male & LHSC Cohort 3 \\
\hline Karen & LHArchiveC23 & 1974 & Female & LHSC Cohort 3 \\
\hline Cathy & LHArchiveC24 & 1974 & Female & LHSC Cohort 3 \\
\hline Rachel & LHArchiveC25 & 1972 & Female & LHSC Cohort 3 \\
\hline Janine & LHArchiveC26 & 1969 & Female & LHSC Cohort 3 \\
\hline Angela & LHArchiveC27 & 1965 & Female & LHSC Cohort 3 \\
\hline Maurice & LHArchiveC32 & 1966 & Male & LHSC Cohort 3 \\
\hline Juliette & LHArchiveC37 & 1973 & Female & LHSC Cohort 3 \\
\hline Shirley & GUI001 & 1999 & Female & GUI, wave 1 at 9 years \\
\hline Daniel & GUI002 & 1999 & Male & GUI, wave 1 at 9 years \\
\hline Emmanuelle & GUI003 & 1999 & Female & GUI, wave 1 at 9 years \\
\hline Mathew & GUI004 & 1999 & Male & GUI, wave 1 at 9 years \\
\hline Avril & GUI005 & 1999 & Female & GUI, wave 1 at 9 years \\
\hline Billy & GUI006 & 1999 & Male & GUI, wave 1 at 9 years \\
\hline Eoin & GUI007 & 1999 & Male & GUI, wave 1 at 9 years \\
\hline Jonathon & GUI008 & 1999 & Male & GUI, wave 1 at 9 years \\
\hline Daniel & GUI0010 & 1999 & Male & GUI, wave 1 at 9 years \\
\hline Luke & GUI0011 & 1999 & Male & GUI, wave 1 at 9 years \\
\hline Ronald & GUI0012 & 1999 & Male & GUI, wave 1 at 9 years \\
\hline Richard & GUI0013 & 1999 & Male & GUI, wave 1 at 9 years \\
\hline Sarah & GUI0014 & 1999 & Female & GUI, wave 1 at 9 years \\
\hline Melissa & GUI0015 & 1999 & Female & GUI, wave 1 at 9 years \\
\hline Debbie & GUI0016 & 1999 & Female & GUI, wave 1 at 9 years \\
\hline Nicola & GUIO017 & 1999 & Female & GUI, wave 1 at 9 years \\
\hline Audrey & GUI0018 & 1999 & Female & GUI, wave 1 at 9 years \\
\hline Audrina & GUI0019 & 1999 & Female & GUI, wave 1 at 9 years \\
\hline Amelie & GUI0020 & 1999 & Female & GUI, wave 1 at 9 years \\
\hline Louise & GUI0021 & 1999 & Female & GUI, wave 1 at 9 years \\
\hline Ruby & GUI0022 & 1999 & Female & GUI, wave 1 at 9 years \\
\hline Frank & GUIO023 & 1999 & Male & GUI, wave 1 at 9 years \\
\hline Christine & GUI0024 & 1999 & Female & GUI, wave 1 at 9 years \\
\hline
\end{tabular}




\begin{tabular}{|c|c|c|c|c|}
\hline $\begin{array}{l}\text { Participant's } \\
\text { pseudonym }\end{array}$ & Archive ID number & $\begin{array}{l}\text { Year of } \\
\text { birth }\end{array}$ & Gender & Collection \\
\hline Sam & GUI0025 & 1999 & Male & GUI, wave 1 at 9 years \\
\hline Fiona & GUI0026 & 1999 & Female & GUI, wave 1 at 9 years \\
\hline Robert & GUI0027 & 1999 & Male & GUI, wave 1 at 9 years \\
\hline Henry & GUIO028 & 1999 & Male & GUI, wave 1 at 9 years \\
\hline Mary & GUI0029 & 1999 & Female & GUI, wave 1 at 9 years \\
\hline Declan & GUI0030 & 1999 & Male & GUI, wave 1 at 9 years \\
\hline Sam & GUI0031 & 1999 & Female & GUI, wave 1 at 9 years \\
\hline Ben & GUI0032 & 1999 & Male & GUI, wave 1 at 9 years \\
\hline Tina & GUI0033 & 1999 & Female & GUI, wave 1 at 9 years \\
\hline Charlotte & GUI0034 & 1999 & Female & GUI, wave 1 at 9 years \\
\hline Gemma & GUI0035 & 1999 & Female & GUI, wave 1 at 9 years \\
\hline Wendy & GUI0036 & 1999 & Female & GUI, wave 1 at 9 years \\
\hline Charlotte & GUI0037 & 1999 & Female & GUI, wave 1 at 9 years \\
\hline Damien & GUI0038 & 1999 & Male & GUI, wave 1 at 9 years \\
\hline Jason & GUI0039 & 1999 & Male & GUI, wave 1 at 9 years \\
\hline Mary & GUI0040 & 1999 & Female & GUI, wave 1 at 9 years \\
\hline Gavin & GUI0041 & 1999 & Male & GUI, wave 1 at 9 years \\
\hline Bill & GUI0042 & 1999 & Male & GUI, wave 1 at 9 years \\
\hline Helen & GUI0043 & 1999 & Female & GUI, wave 1 at 9 years \\
\hline Rachel & GUI0044 & 1999 & Female & GUI, wave 1 at 9 years \\
\hline Sam & GUI0045 & 1999 & Male & GUI, wave 1 at 9 years \\
\hline Dennis & GUI0046 & 1999 & Male & GUI, wave 1 at 9 years \\
\hline Eoin & GUI0047 & 1999 & Male & GUI, wave 1 at 9 years \\
\hline Naomi & GUI0048 & 1999 & Female & GUI, wave 1 at 9 years \\
\hline Karen & GUI0049 & 1999 & Female & GUI, wave 1 at 9 years \\
\hline Paul & GUI0050 & 1999 & Male & GUI, wave 1 at 9 years \\
\hline Damien & GUI0051 & 1999 & Male & GUI, wave 1 at 9 years \\
\hline Peter & GUI0052 & 1999 & Male & GUI, wave 1 at 9 years \\
\hline David & GUI0053 & 1999 & Male & GUI, wave 1 at 9 years \\
\hline Scott & GUI0054 & 1999 & Male & GUI, wave 1 at 9 years \\
\hline Susan & GUI0055 & 1999 & Female & GUI, wave 1 at 9 years \\
\hline Jackie & GUI0056 & 1999 & Female & GUI, wave 1 at 9 years \\
\hline Damien & GUI0057 & 1999 & Male & GUI, wave 1 at 9 years \\
\hline Jeff & GUI0058 & 1999 & Male & GUI, wave 1 at 9 years \\
\hline
\end{tabular}




\begin{tabular}{|c|c|c|c|c|}
\hline $\begin{array}{l}\text { Participant's } \\
\text { pseudonym }\end{array}$ & Archive ID number & $\begin{array}{l}\text { Year of } \\
\text { birth }\end{array}$ & Gender & Collection \\
\hline Dylan & GUI0059 & 1999 & Male & GUI, wave 1 at 9 years \\
\hline Barry & GUI0060 & 1999 & Male & GUI, wave 1 at 9 years \\
\hline Laura & GUI0061 & 1999 & Female & GUI, wave 1 at 9 years \\
\hline Carly & GUI0062 & 1999 & Female & GUI, wave 1 at 9 years \\
\hline John & GUI0063 & 1999 & Male & GUI, wave 1 at 9 years \\
\hline Nick & GUI0064 & 1999 & Male & GUI, wave 1 at 9 years \\
\hline Gillian & GUI0065 & 1999 & Female & GUI, wave 1 at 9 years \\
\hline Sebastian & GUI0066 & 1999 & Male & GUI, wave 1 at 9 years \\
\hline Roisin & GUI0067 & 1999 & Female & GUI, wave 1 at 9 years \\
\hline Zenia & GUI0068 & 1999 & Female & GUI, wave 1 at 9 years \\
\hline Martin & GUI0069 & 1999 & Male & GUI, wave 1 at 9 years \\
\hline Sean & GUI0070 & 1999 & Male & GUI, wave 1 at 9 years \\
\hline Sally & GUI0071 & 1999 & Female & GUI, wave 1 at 9 years \\
\hline Amy & GUI0072 & 1999 & Female & GUI, wave 1 at 9 years \\
\hline Sarah & GUI0073 & 1999 & Female & GUI, wave 1 at 9 years \\
\hline Mark & GUI0074 & 1999 & Male & GUI, wave 1 at 9 years \\
\hline Ian & GUIO075 & 1999 & Male & GUI, wave 1 at 9 years \\
\hline Sophie & GUI0076 & 1999 & Female & GUI, wave 1 at 9 years \\
\hline Paula & GUI0077 & 1999 & Female & GUI, wave 1 at 9 years \\
\hline Simon & GUIO078 & 1999 & Male & GUI, wave 1 at 9 years \\
\hline Vicky & GUI0079 & 1999 & Female & GUI, wave 1 at 9 years \\
\hline Rachel & GUIO080 & 1999 & Female & GUI, wave 1 at 9 years \\
\hline Taylor & GUI0081 & 1999 & Male & GUI, wave 1 at 9 years \\
\hline Colm & GUI0082 & 1999 & Male & GUI, wave 1 at 9 years \\
\hline Leona & GUI0083 & 1999 & Female & GUI, wave 1 at 9 years \\
\hline Daniel & GUIO084 & 1999 & Male & GUI, wave 1 at 9 years \\
\hline Damien & GUI0085 & 1999 & Male & GUI, wave 1 at 9 years \\
\hline Graham & GUI0086 & 1999 & Male & GUI, wave 1 at 9 years \\
\hline Tania & GUI0087 & 1999 & Female & GUI, wave 1 at 9 years \\
\hline Erika & GUIO088 & 1999 & Female & GUI, wave 1 at 9 years \\
\hline Donal & GUI0089 & 1999 & Male & GUI, wave 1 at 9 years \\
\hline Maura & GUI0090 & 1999 & Female & GUI, wave 1 at 9 years \\
\hline Paul & GUI0091 & 1999 & Male & GUI, wave 1 at 9 years \\
\hline Etain & GUI0092 & 1999 & Female & GUI, wave 1 at 9 years \\
\hline
\end{tabular}




\begin{tabular}{|c|c|c|c|c|}
\hline $\begin{array}{l}\text { Participant's } \\
\text { pseudonym }\end{array}$ & Archive ID number & $\begin{array}{l}\text { Year of } \\
\text { birth }\end{array}$ & Gender & Collection \\
\hline Peter & GUI0093 & 1999 & Male & GUI, wave 1 at 9 years \\
\hline Arthur & GUI0094 & 1999 & Male & GUI, wave 1 at 9 years \\
\hline Rory & GUI0095 & 1999 & Male & GUI, wave 1 at 9 years \\
\hline Paul & GUI0096 & 1999 & Male & GUI, wave 1 at 9 years \\
\hline Daniel & GUI0097 & 1999 & Male & GUI, wave 1 at 9 years \\
\hline Wesley & GUI0098 & 1999 & Male & GUI, wave 1 at 9 years \\
\hline Ann & GUI0099 & 1999 & Female & GUI, wave 1 at 9 years \\
\hline Noah & GUI0110 & 1999 & Male & GUI, wave 1 at 9 years \\
\hline Sean & GUI0101 & 1999 & Male & GUI, wave 1 at 9 years \\
\hline Jamie & GUI0102 & 1999 & Female & GUI, wave 1 at 9 years \\
\hline Kenny & GUI0103 & 1999 & Male & GUI, wave 1 at 9 years \\
\hline Damien & GUI0104 & 1999 & Male & GUI, wave 1 at 9 years \\
\hline Sarah & GUI0105 & 1999 & Female & GUI, wave 1 at 9 years \\
\hline Sandra & GUI0106 & 1999 & Female & GUI, wave 1 at 9 years \\
\hline Heidi & GUI0107 & 1999 & Female & GUI, wave 1 at 9 years \\
\hline Daniel & GUI0108 & 1999 & Male & GUI, wave 1 at 9 years \\
\hline Alan & GUI109 & 1999 & Male & GUI, wave 1 at 9 years \\
\hline John & GUI0110 & 1999 & Male & GUI, wave 1 at 9 years \\
\hline Roisin & GUI0111 & 1999 & Female & GUI, wave 1 at 9 years \\
\hline Rhona & GUI0112 & 1999 & Female & GUI, wave 1 at 9 years \\
\hline Freddy & GUI0113 & 1999 & Male & GUI, wave 1 at 9 years \\
\hline Lizzy & GUI0114 & 1999 & Female & GUI, wave 1 at 9 years \\
\hline Olivia & GUI0115 & 1999 & Female & GUI, wave 1 at 9 years \\
\hline Tiffany & GUI0116 & 1999 & Female & GUI, wave 1 at 9 years \\
\hline Simon & GUI0117 & 1999 & Male & GUI, wave 1 at 9 years \\
\hline
\end{tabular}

